\title{
Duplexsonografie der tiefen Beinvenenthrombose
}

Marie-Luise Valentin, Robert Clemens, Christoph Thalhammer

Die farbkodierte Duplexsonografie ist die Untersuchungsmethode der Wahl bei Verdacht auf eine tiefe Beinvenenthrombose. In der Hand eines erfahrenen Untersuchers kann eine Thrombose sonografisch bestätigt oder ausgeschlossen werden, aber auch nicht vaskuläre Differenzialdiagnosen werden erfasst. Lesen Sie hier, wie eine systematische Untersuchung der Beinvenen abläuft.

Inzidenz | Bei einer venösen Thrombose handelt es sich um eine partielle oder vollständige Verlegung der tiefen Leit- oder Muskelvenen. Die tiefe Venenthrombose (TVT) der unteren Extremität ist mit einer jährlichen Inzidenz von etwa 1-3:1000 eine häufige Erkrankung. Die wichtigste, lebensbedrohliche Komplikation ist die Lungenembolie, daher ist ein Verdacht auf eine Thrombose eine Notfallsituation und sollte zeitnah abgeklärt werden.

Indikationen | Die Patienten präsentieren sich mit einseitiger Schwellung, Schmerzen, oder Verfärbung der unteren Extremität. Trotz verschiedener klinischen Risiko-Scores und der Verwendung des D-Dimere-Tests ist die Anamnese und die klinische Untersuchung allein nicht geeignet, eine TVT zu diagnostizieren bzw. auszuschließen. Die Ultraschalldiagnostik ist ein einfaches, kostengünstiges und schnell verfügbares Verfahren zur Bestätigung oder Ausschluss der Verdachtsdiagnose. Die Kompressionssonografie zeigt sowohl eine hohe Sensitivität als auch hohe Spezifität und ist sehr zuverlässig in geübten Händen [1]. Komplikationen gibt es keine, allerdings kann die Kompression des geschwollenen Beins schmerzhaft sein.

Ultraschallgerät | Für die Untersuchung der Beckenvenen verwendet man einen Sektorschallkopf mit einer niedrigen Sendefrequenz (z.B.
3,5-5 MHz). Für die Untersuchung der Beinvenen wird in der Regel ein Linearschallkopf mit einer mittleren Frequenz (z.B. 5-9 MHz) eingesetzt. Heutzutage verfügen alle modernen Ultraschallgeräte über einen Farbdoppler-Modus und die Möglichkeit einer Doppler-Spektralanalyse. Bei frischen Wunden, wie z.B. Operationsnarben im Untersuchungsbereich, muss ein steriler Schallkopfüberzug verwendet werden. Wir empfehlen die grosszügige Verwendung von ausreichend viel Ultraschallgel.

\section{B-Mode Ultraschall}

Kompressionssonografie | Um eine TVT auszuschließen, ist prinzipiell eine komplette Kompressionssonografie (KKS) im Schwarz-WeißBild (B-Mode) ausreichend [2]. Eine Vene lässt sich, im Gegensatz zur parallel verlaufenden Arterie, mit relativ wenig Druck vollständig komprimieren ( $\triangleright$ Abb. 1). Bei der KKS werden sämtliche Beinvenen im Verlauf komprimiert - eine nicht komprimierbare Vene ist praktisch gleichbedeutend mit einer Thrombose. Die KKS wird nur im Querschnitt durchgeführt. Zu Dokumentationszwecken kann der Zwei-Bild-Modus verwendet werden, bei der links die Vene ohne Kompression und rechts im komprimierten $\mathrm{Zu}$ stand zu sehen ist.

Abb. 1 (a) V. femoralis im Querschnitt (Pfeil) und (b) nach Kompression (nicht mehr sichtbar). Die Arterie bleibt darstellbar (Pfeil).
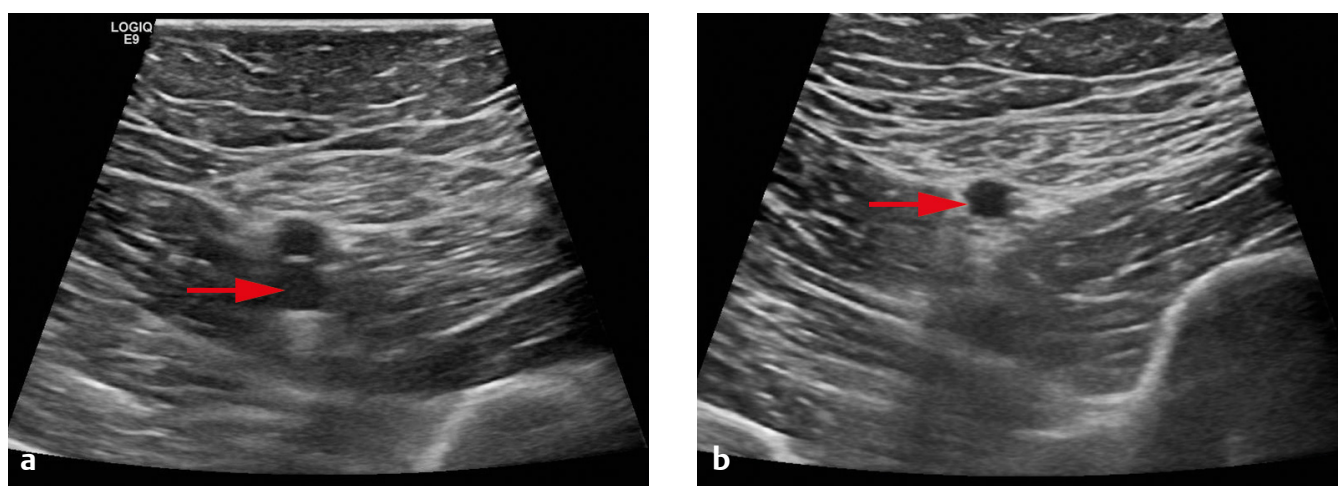
Dokumentation | Folgende Venenabschnitte sollten bei einer vollständigen Untersuchung dokumentiert werden:

- V. femoralis communis

- V.profunda femoris

- V. femoralis (früher: V. femoralis superficialis)

- V. poplitea

- Vv. tibiales posteriores

- (Vv. tibialis anteriores)

- Vv. fibulares

- Muskelvenen (M. gastrocnemius, M. soleus)

\section{Farbdoppler und Doppler- Spektralanalyse}

Farbdoppler I Der farbkodierte Doppler-Modus kann hilfreich sein beim Auffinden von Gefäßen, z.B. der Mündung der V. profunda femoris ( Abb. 2). Da im venösen System relativ langsame Geschwindigkeiten vorliegen, muss die Pulsrepetitionsfrequenz (PRF) entsprechend niedrig eingestellt werden. Die Farbbox sollte möglichst klein eingestellt werden und entsprechend der Verlaufsrichtung des Gefäßes gekippt sein, um einen möglichst kleinen Winkel des Farbdopplers auf das Gefäß zu erhalten. Als weitere, neue Methode kann die B-Flow-Technik sehr langsame Geschwindigkeiten winkelunabhängig sehr schön darstellen ( Abb. 6c).

Doppler-Spektralanalyse | Die Doppler-Spektralanalyse dient zur Dokumentation des venösen Flussmusters, das physiologischerweise kardial und atemmoduliert ist ( Abb. $\mathbf{3}$ a). Eine Seitendifferenz des Flussmusters in der V. femoralis communis mit einem einseitig pathologischen Fluss kann ein wichtiger Hinweis auf eine iliakale Obstruktion sein ( Abb. 3 b).

\section{Untersuchungsablauf}

Allgemeines | Der Patient wird zur Untersuchung der Becken-Beinvenen zunächst in Rückenlage gebracht. Der wohltemperierte Raum sollte abge-

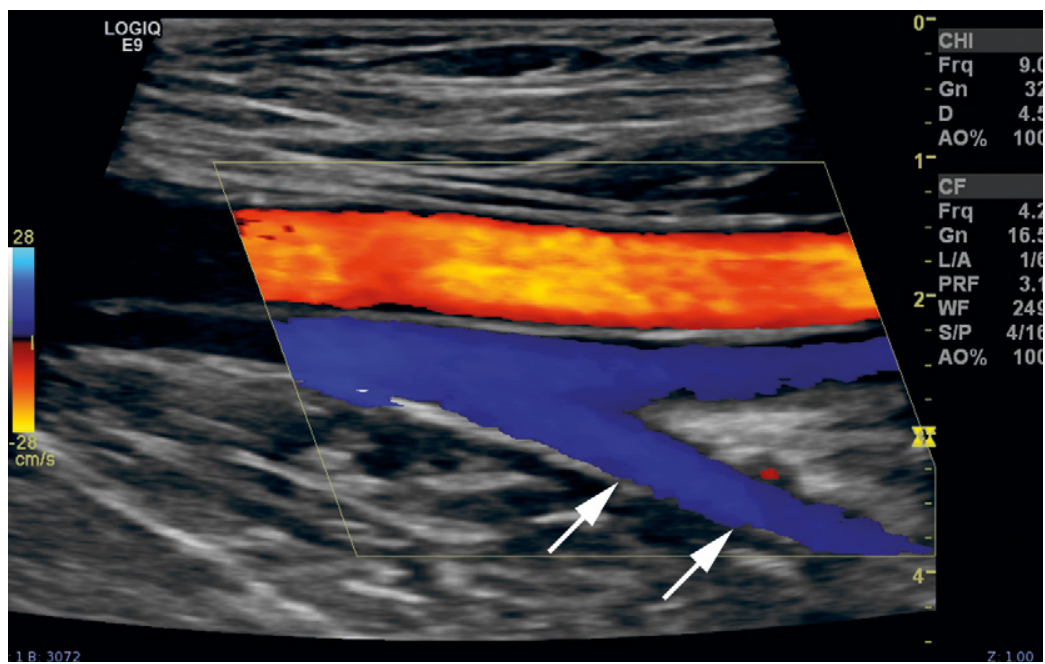

Abb. 2 Konfluens der V. femoralis mit der

dunkelt sein. Für eine komplette Untersuchung eines Beins ist mit einem Zeitaufwand von rund V. profunda femoris, die aus der Tiefe mündet (Pfeile).

Schritt 1 | Die Untersuchung beginnt in der Leiste im Querschnitt mit dem Aufsuchen des Gefäßbündels im B-Mode. Die medial der A. femoralis communis gelegene $\mathrm{V}$. femoralis communis wird nun durch Druck mit dem Schallkopf komprimiert.

- Ist die Vene vollständig komprimierbar?

- Lassen sich echogene Veränderungen im Venenlumen darstellen?

Schritt 2 | Als nächstes wird die V. femoralis communis im Längsschnitt dargestellt. Die Venenwand ist physiologischerweise ganz zart, im Gegensatz zur Arterienwand, die deutlich echoreicher zur Darstellung kommt. Das Lumen der Vene ist bei korrekter Einstellung schwarz und zeigt keine Binnenechos.

Schritt 3 | Im nächsten Schritt wird die V. femoralis im Farbdoppler dargestellt. Die Farbe sollte das Gefäß vollständig ausfüllen.

- Lassen sich umspülte Thromben darstellen?

- Besteht eine Flussmodulation?

Abb. 3 (a) normale Doppler-Spektralanalyse der V. femoralis communis mit kardial und atemmoduliertem Spontanfluss; (b) pathologische Doppler-Spektralanalyse mit bandförmigem nicht moduliertem Fluss als Hinweis auf eine mögliche iliakale Thrombose
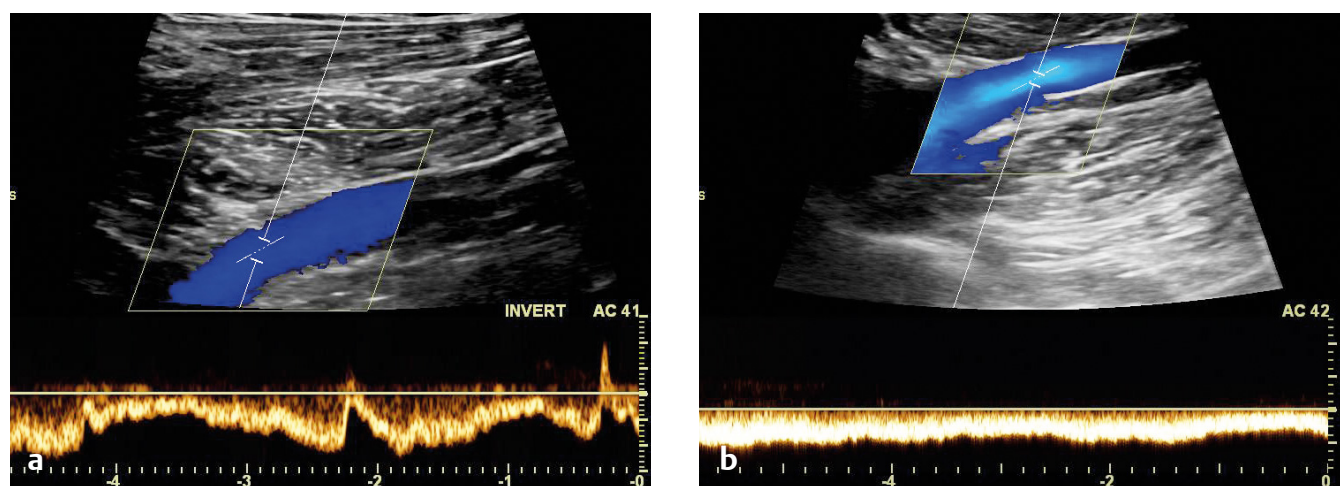


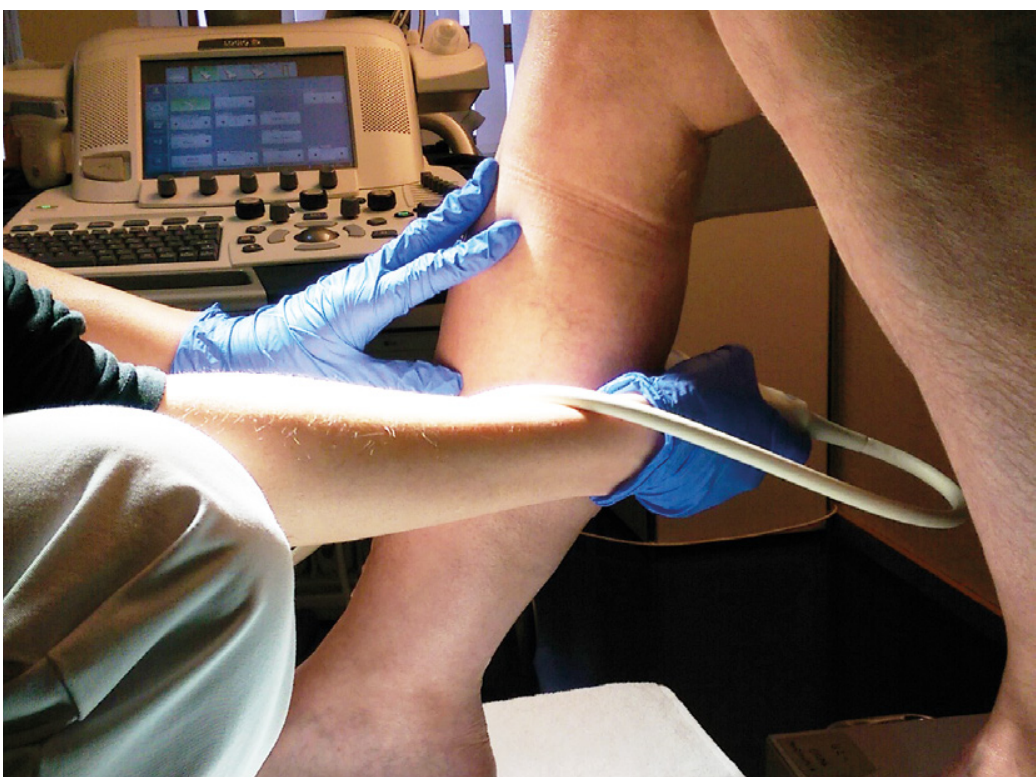

Abb.4 Untersuchung der Unterschenkelvenen im Sitzen: Das betroffene Bein steht nach vorne, das Gegenbein nach hinten.

Schritt 4 | Im nächsten Schritt erfolgt die Ableitung eines Dopplersignals. Venen weisen typische Flussmuster aufgrund der Modulation durch Atmung und Herzschlag auf $(\bullet$ Abb. 3a). Das Flussmuster zeigt einen größtenteils negativen Fluss. Ein bandförmiges, nicht moduliertes Flussmuster weist auf eine proximale Abflussbehinderung hin $(\triangleright$ Abb. $\mathbf{3 b}$ b). Das Flussmuster muss stets im Seitenvergleich abgeleitet werden und weist im Normalfall keine wesentlichen Seitenunterschiede auf. So kann indirekt die Durchgängigkeit der Beckenvenen überprüft werden.

- kardiale Modulation?

- Atemvariabilität?

- Seitenvergleich?

Schritt 5 | Es bewährt sich, die V. profunda femoris im Farbdoppler durch langsames entlangfahren der V. femoralis communis im Längsschnitt aufzusuchen $(\triangleright$ Abb. 2 ).
Die V. profunda femoris lässt sich meist nur ganz proximal darstellen. Eine isolierte TVT dieser Vene ist sehr selten und dann als atypische Thrombose ein Hinweis auf Malignom.

Schritt 6 | Die Darstellung der Venen des, wenn möglich leicht nach aussen rotierten, Oberschenkels erfolgt im Querschnitt am liegenden Patienten. Dazu wird in regelmässigen sehr kurzen $\mathrm{Ab}$ ständen die Vene komprimiert und wie oben beschrieben dokumentiert.

\section{Distal zieht die V. femoralis durch den} Adduktorenkanal in die Tiefe. Eine vollständige Kompression ist hier oft nicht allein mit dem Schallkopf möglich. Es hat sich bewährt, mit der Hand die Muskulatur in Richtung Schallkopf zu drücken.

Schritt 7 | Die V. poplitea und Unterschenkelvenen werden im Sitzen untersucht, da die Venen bei besserer Füllung leichter abgegrenzt werden können ( Abb. 4). Die Vena poplitea wird in gleicher Weise wie die V. femoralis communis untersucht. Zunächst erfolgen die Beurteilung im B-Mode und die Kompression, dann im Längsschnitt mit Farbdoppler und schließlich Ableitung eines Dopplersignals. Aufgrund der Entfernung zum Thorax ist das Flusssignal nicht immer spontan moduliert. Zum Nachweis der Durchgängigkeit wird im Dopplermodus die Wade manuell komprimiert, was zu einem kurzen schnellen Flussnachweis führt.

Schritt 8 | Die Unterschenkelvenen stellen sich von dorsal und leicht medial am besten dar. Als Orientierung dienen die zwei Knochenkanten der Tibia und der Fibula $(\checkmark$ Abb. 5 a). Man sollte versuchen, diese nebeneinander abzubilden, die Gefäßbündel liegen dann schön in der Nähe der Knochenkanten. Auch am Unterschenkel erfolgt

Abb. 5 Unterschenkelvenen im Querschnitt. (a) A. tibialis posterior mit zwei Begleitvenen dorsal der Tibia (Pfeil) und A. fibularis mit zwei Begleitvenen medial der Fibula; (b) Unterschenkelvenen während Kompression: es stellen sich nur noch die entsprechenden Arterien dar.
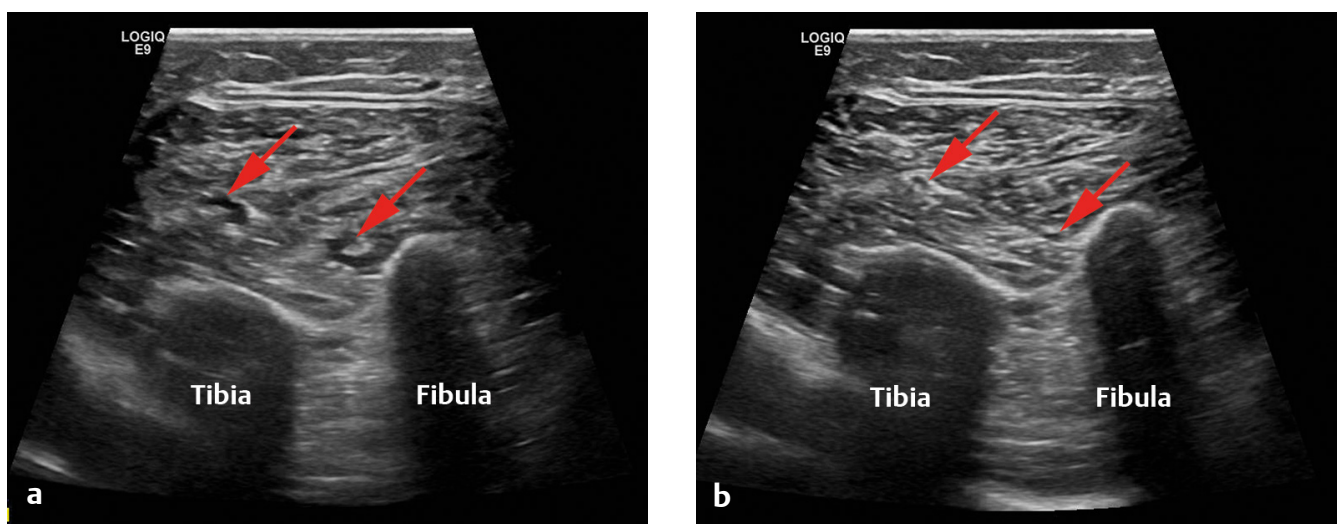

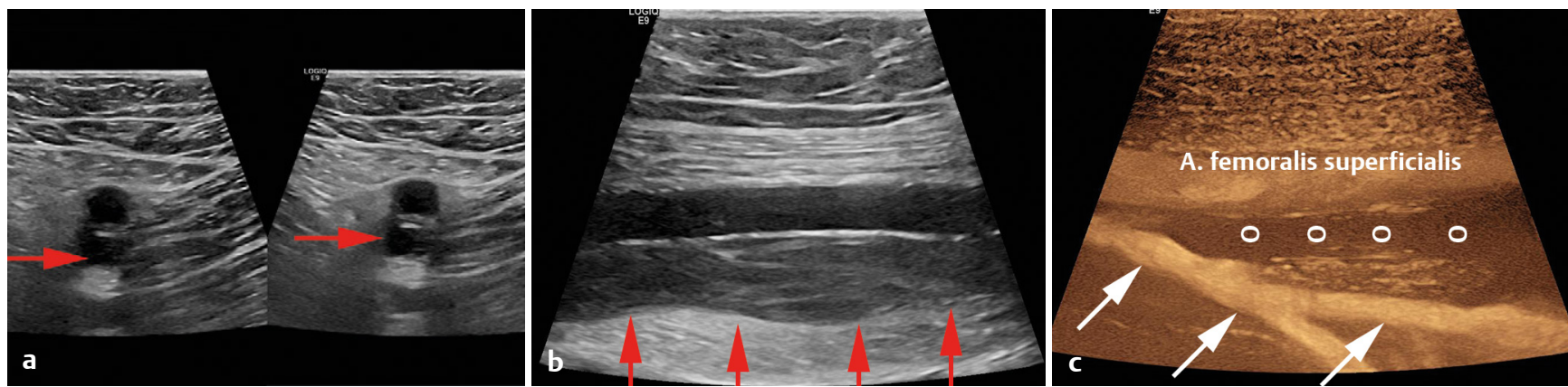

Abb. 6 (a) V. femoralis (Pfeil) im Querschnitt links ohne, rechts mit Kompression bei tiefer Venenthrombose; (b) V. femoralis (Pfeile) im Längsschnitt: aufgetriebene Vene (im Vergleich zur Arterie) mit echogenem Material gefüllt; (c) B-Flow-Sonografie der V. profunda femoris (Pfeile) mit schöner Flussdarstellung und Thrombose der V. femoralis ohne Fluss im B-Flow (Kreise).

die schrittweise KKS mit Dokumentation der vollständigen Komprimierbarkeit $(\triangleright$ Abb. 5 b)

Bei schwer darstellbaren Venen kann der Farbdoppler (oder der B-Flow) zur Hilfe genommen werden. Man fokussiert auf die Unterschenkelarterie und provoziert durch distale Kompression der Wade meist ein gutes Farbsignal.

Muskelvenen | Die Muskelvenen des M. gastrocnemius und des $M$. soleus werden ebenfalls von dorsal proximal aufgesucht und dann sowohl im Verlauf des medialen als auch lateralen Kopfes nach distal untersucht. Die V. tibialis anterior wird nur bei expliziten Fragestellungen und dortiger Schmerzlokalisation untersucht.

Eine isolierte Thrombose der Vv. tibiales anteriores ist eine Rarität.

Tipp | Fragen Sie den Patienten nach Schmerzpunkten und untersuchen Sie dort gezielt.

\section{Sonografische Thrombosezeichen}

- Inkompressibilität ( Abb. 6a)

- keine Atemvariabilität des Venenlumens

Abb. 7 Fehldiagnose einer Thrombose der distalen V. femoralis, die nicht komprimierbar ist, da mit dem Schallkopf gegen den Knochen gedrückt wurde (Pfeile).

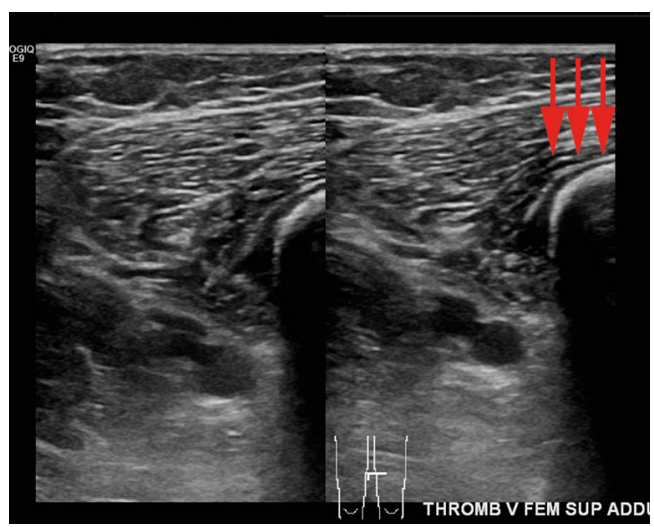

- erhöhter Durchmessers im Vergleich zur gesunden Vene resp. zur Arterie ( Abb. 6b)

- Intravasale echogene Binnenstruktur ( Abb. 6b)

- bei kompletter Thrombose ist im gesamten Venenlumen keine Farbe nachweisbar (Abb. 6c)

- bei partieller Thrombosierung ist im durchflossenen Restlumen Farbe nachweisbar

- bei kompletter Okklusion ist kein Dopplersignal ableitbar

- bandförmiges, nicht moduliertes Flussmuster in den nicht thrombosierten Abschnitten (Abb. 3b)

Pitfall | Kompression mit dem Schallkopf auf den Knochen bringt die daneben liegende Venen nicht zum Kollabieren ( Abb. 7).

\section{Häufige Differenzialdiagnosen}

- Bakerzyste: sackförmige, meist echofreie, scharf begrenzte Struktur ausgehend von dem medialen Kniegelenksspalt, keine Perfusion nachweisbar

- Hämatom: Kompression der Vene von außen (z.B. Serom iliakal nach Nierentransplantation, Schwangerschaft, abdominelle Tumoren)

- arterielle Aneurysmata: am häufigsten betroffen ist die A. poplitea

- vaskuläre Malformationen

\section{Konsequenz für Klinik und Praxis}

- In der Hand eines erfahrenen Untersuchers ist die Duplexsonografie der Beinvenen Methode der Wahl zur Sicherung und zum Ausschluss einer tiefen Beinvenenthrombose.

- Auch nicht-vaskuläre Ursachen der Beinschwellung sind der Sonografie gut zugänglich.

Interessenkonflikt

Die Autoren geben an, dass kein Interessenkonflikt besteht.

Vollständiges Literaturverzeichnis unter http://dx.doi.org/10.1055/s-0042-108930

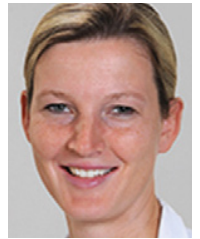

Dr. med. Marie-Luise Valentin

ist Oberärztin an der Klinik für Angiologie des UniversitätsSpitals Zürich.

marie-luise.valentin@usz.ch

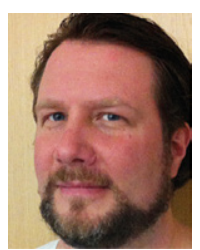

Dr. med. Robert Clemens ist Oberarzt an der Klinik für Angiologie des UniversitätsSpitals Zürich.

robert.clemens@usz.ch

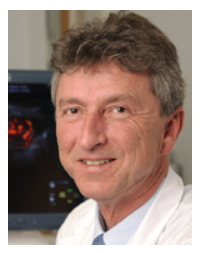

PD Dr. med. Christoph Thalhammer ist leitender Arzt an der Klinik für Angiologie des UniversitätsSpitals Zürich und Leiter der Duplexsonografie. Christoph.Thalhammer@ usz.ch

DOI 10.1055/s-0042-108930

Dtsch Med Wochenschr 2016; 141: 946-949

(c) Georg Thieme Verlag KG . Stuttgart · New York. ISSN 0012-0472 


\section{Literatur}

1 Hach-Wunderle V, Kopp I et al. Interdisziplinäre S2k-Leitlinie - Diagnostik und Therapie der Venenthrombose und der Lungenembolie. VASA 2016; 45 (suppl 90): 1-48

2 Amann-Vesti B, Thalhammer C. Hrsg. Kursbuch Doppler- und Duplexsonographie: Ultraschalldiagnostik venöser Gefäßerkrankungen. Stuttgart: Thieme; 2016 